\title{
$S L_{n}(F[x])$ IS NOT BOUNDEDLY GENERATED BY ELEMENTARY MATRICES: EXPLICIT PROOF
}

\author{
IGOR V. EROVENKO
}

\begin{abstract}
Using methods of higher algebraic $K$-theory, van der Kallen proved that $S L_{n}(F[x])$ does not have bounded word length with respect to elementary matrices if the field $F$ has infinite transcendence degree over its prime subfield. We exhibit a short explicit proof of this result by constructing a sequence of matrices with infinitely growing word length. We also use this construction to show that $S L_{n}(\mathbb{Z}[x])$ does not have bounded word length with respect to elementary matrices of "bounded degree".
\end{abstract}

\section{INTRODUCTION}

Let $G$ be an abstract group and $X$ be a set of generators of $G$. Recall that $G$ is said to have bounded word length with respect to $X$ (or to be boundedly generated by $X$ ) if there exists a positive integer $N$ such that every element of $G$ can be represented as a product of at most $N$ elements from $X$. Carter and Keller [1] proved that $S L_{n}(\mathscr{O})$, $n \geqslant 3$, where $\mathscr{O}$ is the ring of integers in an algebraic number field, is boundedly generated by the set of elementary matrices. One might expect that this result can be extended to arbitrary Euclidean rings, however, it turned out that delicate arithmetic techniques involved in the proof of the result in [1] (particularly, Dirichlet's prime number theorem) cannot be bypassed. It was shown by van der Kallen [7] that $S L_{n}(F[x]), n \geqslant 3$, does not have bounded word length with respect to elementary matrices if the field $F$ has infinite transcendence degree over its prime subfield. His proof is of "existence" type and is essentially based on the fact that for such a field $K_{2}(F)$ does not have bounded word length with respect to the Steinberg symbols. The goal of this note is to give an explicit proof of van der Kallen's result by constructing a sequence of matrices from $S L_{n}(F[x])$ with infinitely growing word length.

Since every element in $K_{2}(\mathbb{Q})$ is a single Steinberg symbol [3], methods of algebraic $K$-theory alone are not sufficient to yield the answer in the general case. The question whether $S L_{n}(\mathbb{Q}[x])$ is boundedly generated by elementary matrices is still open. It is also interesting to know the answer for $S L_{n}(\mathbb{Z}[x])$ since it is closely related to Kazhdan's property (T). An affirmative answer would provide first examples of linear Kazhdan groups which are not lattices (see [6] for more details). We use the explicit construction of $₫ 2$ to show that $S L_{n}(\mathbb{Z}[x])$ does not have bounded word length with respect to the elementary matrices of "bounded degree" (see $\$ 3$ for definitions). The idea to use $p$-adic numbers is due to A.S. Rapinchuk.

$$
\text { 2. } S L_{n}(F[x])
$$

For an element $r$ of an arbitrary ring $R$ we let $e_{i j}(r)$ denote the corresponding elementary matrix in $S L_{n}(R)$ : it has 1's on the main diagonal and the only nonzero 
off-diagonal entry is $r$ in position $(i, j)$. Let $\left\{r_{n}\right\}$ be an infinite sequence of independent transcendental elements in $F$ and for $n \geqslant 1$ let

$$
\begin{aligned}
A_{n}(x)= & e_{12}\left(r_{2 n-1} r_{2 n}\right) e_{21}\left(-r_{2 n-1}^{-1} r_{2 n}^{-1}+x r_{2 n-1}^{-1} r_{2 n}^{-1}\right) e_{12}\left(r_{2 n-1} r_{2 n}\right) \\
& \times e_{12}(-1) e_{21}(1-x) e_{12}(-1) \\
& \times\left[e_{12}\left(r_{2 n-1}\right) e_{21}\left(-r_{2 n-1}^{-1}+x r_{2 n-1}^{-1}\right) e_{12}\left(r_{2 n-1}\right) e_{12}(-1) e_{21}(1-x) e_{12}(-1)\right]^{-1} \\
& \times\left[e_{12}\left(r_{2 n}\right) e_{21}\left(-r_{2 n}^{-1}+x r_{2 n}^{-1}\right) e_{12}\left(r_{2 n}\right) e_{12}(-1) e_{21}(1-x) e_{12}(-1)\right]^{-1} .
\end{aligned}
$$

Set

$$
B_{k}(x)=\prod_{n=1}^{k} A_{n}(x) .
$$

Theorem 2.1 The sequence $\left\{B_{k}(x)\right\} \subseteq S L_{n}(F[x])$ does not have bounded word length with respect to elementary matrices.

Proof. Assume the contrary. Suppose that there exists an integer $N>0$ such that for every $k$ there is a presentation of length $\leqslant N$

$$
B_{k}(x)=u_{1}(x) \cdots u_{N}(x)
$$

for some elementary matrices $u_{i}(x)$. (We may assume that all such presentations have length $N$ by inserting matrices $e_{12}(0)$.) Then we obtain the following equality in the Steinberg group $S t_{n}(F[x])$ :

$$
\tilde{B}_{k}(x)=T(x) \tilde{u}_{1}(x) \cdots \tilde{u}_{N}(x),
$$

where $\tilde{B}_{k}(x)$ is obtained by taking canonical lifts of elementary matrices $e_{i j}(*)$ from presentations $(1), \tilde{u}_{i}(x)$ is the canonical lift of the elementary matrix $u_{i}(x)$, and $T(x)$ is an element of $K_{2}(F[x])$.

Next, we use van der Kallen's idea from [7] and specialize (3) at $x=0$ and $x=1$ to obtain equalities

$$
\tilde{B}_{k}(0)=T(0) \tilde{u}_{1}(0) \cdots \tilde{u}_{N}(0)
$$

and

$$
\tilde{B}_{k}(1)=T(1) \tilde{u}_{1}(1) \cdots \tilde{u}_{N}(1)
$$

in the Steinberg group $S t_{n}(F)$. Note that from (1) and (2) we have

$$
\tilde{B}_{k}(0)=\prod_{n=1}^{k}\left\{r_{2 n-1}, r_{2 n}\right\}
$$

where $\left\{r_{2 n-1}, r_{2 n}\right\}$ is a Steinberg symbol, and

$$
\tilde{B}_{k}(1)=x_{12}(*),
$$

where $x_{12}(*)$ is a canonical generator of the Steinberg group $S t_{n}(F)$.

Since $K_{2}(F[x])=K_{2}(F)$ (see [5]), we obtain the following equality in $S t_{n}(F)$ :

$$
x_{12}(-*) \prod_{n=1}^{k}\left\{r_{2 n-1}, r_{2 n}\right\}=\tilde{u}_{N}^{-1}(1) \cdots \tilde{u}_{1}^{-1}(1) \tilde{u}_{1}(0) \cdots \tilde{u}_{N}(0)
$$


which holds for arbitrarily large $k$. Matsumoto's theorem (see, for example, [4]) implies that the sequence consisting of products of Steinberg symbols

$$
\left\{\prod_{n=1}^{k}\left\{r_{2 n-1}, r_{2 n}\right\}\right\}_{k=1}^{\infty}
$$

does not have bounded word length in $K_{2}(F)$ in terms of Steinberg symbols. On the other hand, it follows from Bruhat decomposition for $S t_{n}(F)$ (see, for example, [2]) that this implies that it does not have bounded word length in $S t_{n}(F)$ with respect to the canonical generators (this has been observed by van der Kallen), whence the contradiction.

$$
\text { 3. } S L_{n}(\mathbb{Z}[x])
$$

We begin with the following observation.

Lemma 3.1 $S L_{n}\left(\mathbb{Z}_{p}[x]\right)$ does not have bounded word length with respect to elementary matrices.

Proof. Since $\mathbb{Q}_{p}$ is complete it is uncountable, therefore, has infinite transcendence degree over $\mathbb{Q}$. Let $\left\{r_{n}\right\} \subseteq \mathbb{Q}_{p}$ be a sequence of independent transcendental elements. Write $r_{n}=p^{l_{n}} s_{n}$ with $s_{n} \in \mathbb{Z}_{p}^{\times}$. Using multiplicativity of Steinberg symbols we obtain

$$
\begin{aligned}
\prod_{n=1}^{k}\left\{r_{2 n-1}, r_{2 n}\right\} & =\prod_{n=1}^{k}\left\{r_{2 n-1}, p^{l_{2 n}}\right\}\left\{p^{l_{2 n-1}} s_{2 n-1}, s_{2 n}\right\} \\
& =\prod_{n=1}^{k}\left\{r_{2 n-1}, p\right\}^{l_{2 n}}\left\{p^{l_{2 n-1}, s_{2 n}}\right\}\left\{s_{2 n-1}, s_{2 n}\right\} \\
& =\prod_{n=1}^{k}\left\{r_{2 n-1}^{l_{2 n}}, p\right\}\left\{p, s_{2 n}\right\}^{l_{2 n-1}\left\{s_{2 n-1}, s_{2 n}\right\}} \\
& =\prod_{n=1}^{k}\left\{r_{2 n-1}^{l_{2 n}}, p\right\}\left\{s_{2 n}^{l_{2 n-1}}, p\right\}\left\{s_{2 n-1}, s_{2 n}\right\} \\
& =\left\{\prod_{n=1}^{k} r_{2 n-1}^{l_{2 n}} s_{2 n}^{l_{2 n-1}}, p\right\} \prod_{n=1}^{k}\left\{s_{2 n-1}, s_{2 n}\right\} .
\end{aligned}
$$

Therefore, since the sequence $\left\{\prod_{n=1}^{k}\left\{r_{2 n-1}, r_{2 n}\right\}\right\}$ has infinitely growing word length with respect to Steinberg symbols in $K_{2}\left(\mathbb{Q}_{p}\right)$, the sequence $\left\{\prod_{n=1}^{k}\left\{s_{2 n-1}, s_{2 n}\right\}\right\}$ has the same property.

Next, construct matrices $A_{n}(x)$ and $B_{k}(x)$ as in (1) and (2) but use the sequence $\left\{s_{n}\right\}$ instead of $\left\{r_{n}\right\}$. Since all $s_{n}$ 's are $p$-adic integer units, each elementary matrix in the representation of $A_{n}(x)$ has entries with $p$-adic integer coefficients. We conclude that $B_{k}(x) \in S L_{n}\left(\mathbb{Z}_{p}[x]\right)$ and the proof of Theorem 2.1 implies that the sequence $\left\{B_{k}(x)\right\}$ does not have bounded word length in $S L_{n}\left(\mathbb{Z}_{p}[x]\right)$.

Now we will show that $S L_{n}(\mathbb{Z}[x])$ does not have bounded word length with respect to elementary matrices with entries of "bounded degree". First, we make the 
following convention. If $A$ is a nonzero polynomial matrix, we define the degree of $A$ to be the maximum of the degrees of its nonzero polynomial entries.

Proposition 3.2 There is no constant $N$ such that every matrix $A \in S L_{n}(\mathbb{Z}[x])$ can be written as a product of at most $N$ elementary matrices each of which is of degree $\leqslant \delta(d)$, where $d$ is the degree of $A$ and $\delta: \mathbb{N} \rightarrow \mathbb{N}$ is a nondecreasing function.

Proof. Assume the contrary. By Lemma 3.1 we can find a matrix $A \in S L_{n}\left(\mathbb{Z}_{p}[x]\right)$ which cannot be written as a product of $N$ elementary matrices. Let

$$
A=\prod_{k=1}^{s} e_{i_{k} j_{k}}\left(f_{k}(x)\right)
$$

be the shortest possible presentation of $A$ as a product of elementary matrices from $S L_{n}\left(\mathbb{Z}_{p}[x]\right), s>N$. Our goal is to construct a sequence of matrices $\left\{A_{t}\right\} \subseteq S L_{n}(\mathbb{Z}[x])$ converging to $A$.

For each elementary matrix $e_{i j}(f(x))$ in the presentation (4) we construct a sequence of elementary matrices $\left\{u^{(t)}\right\} \subseteq S L_{n}(\mathbb{Z}[x])$ as follows. Write

$$
f(x)=a_{0}+a_{1} x+\cdots+a_{m} x^{m}
$$

with $a_{i} \in \mathbb{Z}_{p}, 0 \leqslant i \leqslant m$, where

$$
a_{i}=\sum_{l=0}^{\infty} a_{i, l} p^{l}, \quad 0 \leqslant a_{i, l} \leqslant p-1,
$$

and define

$$
u^{(t)}=e_{i j}\left(f^{(t)}(x)\right)
$$

where

$$
f^{(t)}(x)=a_{0}^{(t)}+a_{1}^{(t)} x+\cdots+a_{m}^{(t)} x^{m} \quad \text { and } \quad a_{i}^{(t)}=\sum_{l=0}^{t} a_{i, l} p^{l} \in \mathbb{Z} .
$$

Clearly, $u^{(t)} \in S L_{n}(\mathbb{Z}[x])$ and

$$
\lim _{t \rightarrow \infty} u^{(t)}=e_{i j}(f(x)) .
$$

If we set

$$
A_{t}=\prod_{k=1}^{s} u_{k}^{(t)}
$$

where the sequence $\left\{u_{k}^{(t)}\right\}_{t=1}^{\infty}$ is constructed as above to converge to $e_{i_{k} j_{k}}\left(f_{k}(x)\right), 1 \leqslant$ $k \leqslant s$, then $A_{t} \in S L_{n}(\mathbb{Z}[x])$ for all $t$ and the sequence $\left\{A_{t}\right\}$ converges to $A$. Moreover, we observe that the degrees of $A_{t}$ 's are uniformly bounded by $\sum_{k=1}^{s} \operatorname{deg} f_{k}$ (in this sum we, of course, include only those $k$ for which the polynomial $f_{k}(x)$ is not zero).

By our assumption for each matrix $A_{t}$ there is a presentation

$$
A_{t}=\prod_{k=1}^{s_{t}} e_{i_{k} j_{k}}\left(g_{k}^{(t)}(x)\right)
$$

with $s_{t} \leqslant N, g_{k}^{(t)}(x) \in \mathbb{Z}[x]$, and $\operatorname{deg} g_{k}^{(t)} \leqslant M$ for $M=\delta\left(\sum_{k=1}^{s} \operatorname{deg} f_{k}\right), t=1,2, \ldots$ 
Enlarging $N$ we can always assume that the order of the occurrence of elementary matrices in (5) is fixed, and, in particular, that $s_{t}=N$ for all $t$. For this we just insert $e_{i j}(0)$ whenever the corresponding matrix is missing. Thus, without loss of generality we may assume that the indices $i_{k}$ and $j_{k}$ in (5) are the same for all $t$.

Write

$$
g_{1}^{(t)}(x)=\sum_{l=0}^{M} b_{l}^{(t)} x^{l}, \quad t=1,2, \ldots
$$

for some $b_{l}^{(t)} \in \mathbb{Z}$. Since $\mathbb{Z}$ is compact in $\mathbb{Z}_{p}$, the sequence $\left\{b_{0}^{(t)}\right\}$ has a convergent subsequence $\left\{b_{0}^{\left(t_{m}\right)}\right\}$. Say $b_{0}^{\left(t_{m}\right)} \rightarrow b_{0} \in \mathbb{Z}_{p}$.

Next, we pick a subsequence of $\left\{b_{1}^{\left(t_{m}\right)}\right\}$ which converges to some $b_{1} \in \mathbb{Z}_{p}$. Continue in a similar manner by induction to find a sequence of indices $\left\{t_{r}\right\}_{r=1}^{\infty}$ such that each subsequence $\left\{b_{l}^{\left(t_{r}\right)}\right\}$ converges to some $b_{l} \in \mathbb{Z}_{p}, 0 \leqslant l \leqslant M$. The crucial moment here is that the degrees of all $g_{1}^{(t)}$, s are uniformly bounded by $M$.

Observe that

$$
\lim _{r \rightarrow \infty} e_{i_{1} j_{1}}\left(g_{1}^{\left(t_{r}\right)}(x)\right)=e_{i_{1} j_{1}}\left(g_{1}(x)\right)
$$

where

$$
g_{1}(x)=\sum_{l=0}^{M} b_{l} x^{l} \in \mathbb{Z}_{p}[x] .
$$

Next, consider the subsequence $\left\{e_{i_{2} j_{2}}\left(g_{2}^{\left(t_{r}\right)}(x)\right)\right\}_{r=1}^{\infty}$. Arguing as above we can find a subsequence $\left\{t_{r_{m}}\right\}$ of $\left\{t_{r}\right\}$ such that

$$
\lim _{m \rightarrow \infty} e_{i_{2} j_{2}}\left(g_{2}^{\left(t_{r_{m}}\right)}(x)\right)=e_{i_{2} j_{2}}\left(g_{2}(x)\right)
$$

with $g_{2}(x) \in \mathbb{Z}_{p}[x]$

Continue by induction to find a subsequence of indices $\left\{t_{n}\right\}_{n=1}^{\infty}$ with the property that

$$
\lim _{n \rightarrow \infty} e_{i_{k} j_{k}}\left(g_{k}^{\left(t_{n}\right)}(x)\right)=e_{i_{k} j_{k}}\left(g_{k}(x)\right),
$$

for some $g_{k}(x) \in \mathbb{Z}_{p}[x], 1 \leqslant k \leqslant N$. Finally, let

$$
B=\prod_{k=1}^{N} e_{i_{k} j_{k}}\left(g_{k}(x)\right) \text {. }
$$

Then the subsequence $\left\{A_{t_{n}}\right\}$ converges to $B$ which forces $B=A$. But the equality (6) contradicts our choice of $A$, proving the proposition.

\section{REFERENCES}

[1] D. Carter and G. Keller, Bounded elementary generation of $S L_{n}(\mathscr{O})$, Amer. J. Math. 105 (1983), 673-687.

[2] A.J. Hahn and O.T. O’Meara, The Classical Groups and $K$-theory, Springer-Verlag, 1989.

[3] H.W. Lenstra, Jr., $K_{2}$ of a global field consists of symbols, Lecture Notes in Math. 551 (1976), 69-73.

[4] J. Milnor, Introduction to Algebraic $K$-Theory, Princeton University Press, Princeton, New Jersey, 1971.

[5] D. Quillen, Higher algebraic K-theory. I, Lecture Notes in Math. 341 (1973), 85-147. 
[6] Y. Shalom, Bounded generation and Kazhdan's property (T), Inst. Hautes Études Sci. Publ. Math. 90 (1999), 145-168.

[7] W. van der Kallen, $S L_{3}(\mathbb{C}[x])$ does not have bounded word length, Lecture Notes in Math. 966 (1982), 357-361.

Department of Mathematical SCIENCES, University of North Carolina at GreEnsBORO, GREENSBORO NC 27402, USA

E-mail address: igor@uncg. edu 